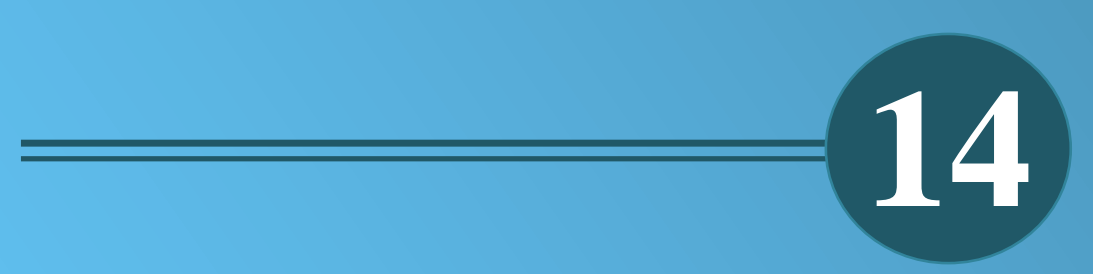

\title{
Selección de árboles semilleros en plantaciones forestales de la provincia de Santa Elena, Ecuador.
}

Juan Valladolid Ontaneda, Ángel León Mejía, Daniel Paredes

Tomalá

Recibido: febrero 2017

Aprobado: mayo 2017 


\title{
Selección de Árboles Semilleros en Plantaciones Forestales de la Península de Santa Elena. Ecuador. \\ Selection of Seed Trees in Forest Plants of the Santa Elena Peninsula. Ecuador.
}

\author{
Juan Valladolid Ontaneda, Ángel León Mejía, Daniel Paredes Tomalá \\ Centro de Investigaciones Agropecuarias \\ Facultad de Ciencias Agrarias \\ Universidad Estatal Península de Santa Elena \\ Campus La Libertad, vía principal Santa Elena - La Libertad \\ La Libertad - Ecuador \\ aleon@upse.edu.ec
}

\begin{abstract}
Resumen
El estudio de selección de árboles semilleros se realizó en las comunas Olón, Dos Mangas y Salanguillo del cantón Santa Elena, provincia de Santa Elena, Ecuador. El objetivo del estudio fue, identificar, caracterizar y seleccionar árboles semilleros de especies maderables con buenas características morfológicas, y que permitan disponer de semillas para trabajos de propagación de especies forestales en viveros con fines de reforestación. El estudio se inicia con la identificación y ubicación de plantaciones forestales de varias especies forestales establecidas entre los años 2000 a 2005 , en las plantaciones se evaluaron las características dasométricas y morfológicas, las que permitieron seleccionar 11 árboles con características morfológicas deseables correspondientes a cinco especies forestales laurel blanco(Cordia alliodora), laurel negro(Cordia megalantha), caoba (Swietenia macrophylla), cedro (Cedrela odorata) y melina (Gmelina arbórea); cada árbol fue georreferenciado utilizando coordenadas UTM Datum WGS 1984 Zona 17M. La cantidad de semillas que pueden producir dichas especies forestales es proyectada por año la cual servirá como referencia para establecer programas de reforestación con materiales propios y adaptados a las condiciones ambientales en la provincia de Santa Elena.
\end{abstract}

Palabras clave: árboles semilleros, árboles padre, forestal, reforestación, dasonomía.

\begin{abstract}
The study of selection of seed trees was carried out in the Olón, Dos Mangas and Salanguillo communes of the canton Santa Elena, Santa Elena province, Ecuador. The objective of the study was to identify, characterize and select seedlings of timber species with good morphological characteristics, and to allow seeds to be used for the propagation of forest species in nurseries for reforestation purposes. The study began with the identification and location of forest plantations of several forest species established between 2000 and 2005, on the plantations, the dasometric and morphological characteristics were evaluated, allowing the selection of 11 trees with desirable morphological characteristics corresponding to five forest species White laurel (Cordia alliodora), black laurel (Cordia megalantha), mahogany (Swietenia macrophylla), cedar (Cedrela odorata) and melina (Gmelina arborea); Each tree was georeferenced using coordinates UTM Datum WGS 1984 Zone 17M. The number of seeds that can produce these forest species is projected per year which will serve as a reference to establish reforestation programs with their own materials and adapted to the environmental conditions in the province of Santa Elena.
\end{abstract}

Keywords: seed tree, parent trees, forest, reforestation, forestry. 


\section{Introducción}

La deforestación en el país ha registrado valores que van desde los años 1990-2000 con un promedio de 89 944 ha/año, mientras que los años 2000-2008 se obtuvo un promedio de 77647 ha/año, para que en los siguientes años como 2008-2012 y se fue reduciendo 65 880 ha/año, por último, la estimación para los años 2013-2018 establece un nivel de 55000 ha/año. Lo cual se considera la base para los siguientes proyectos de reforestaciones en el país. (MAE, 2014).

Los bosques en la región costa son afectados por la ampliación de la frontera agrícola, tala indiscriminada para la obtención de madera, construcciones de viviendas y áreas para ganadería. Mientras que en la sierra la vegetación ha cambiado por nuevos cultivos y viviendas solo se encuentran pequeños sitios con bosques nativos, localizados en las partes con mayores pendientes, donde ya no es posible realizar agricultura, zonas donde se puede observar la erosión por la falta de cobertura vegetal. El Ecuador tiene como proyecto utilizar especies forestales nativas en la reforestación de 26301.47 ha anuales durante 5 años. (MAE, 2013).

En este contexto, los bosques nativos en el Ecuador son importantes para la estabilidad ambiental, calidad y permanencia de las cuencas hidrográficas, que mantiene una variedad en plantas y animales, además contiene productos forestales maderables y no maderables y por ende son capaces de fijar carbono y purificar el aire. (Lojan, 2003).

Sin embargo, en los últimos años, los bosques que se encuentran en la cordillera Chongón-Colonche (Santa Elena) están sometidos a un agresivo proceso de deforestación, siendo sus principales causas la tala de bosque nativo para ampliar la frontera agrícola y la explotación de especies forestales. (CIIFEN, 2014)

La tala de la vegetación limita las posibilidades de recuperación del bosque nativo, lo cual empobrece y disminuye la biodiversidad, dificultando los programas de reforestación, cuya demanda de semilla de alta calidad genética no se encuentra y se utiliza semillas forestales de procedencia desconocida y de baja calidad (Grijalva et al, 2013).

Los recursos genéticos forestales se van perdiendo de modo acelerado por falta de incentivos y conservación. El conocimiento de los recursos genéticos forestales en el Ecuador va en aumento, aunque, son mínimos los estudios de las instituciones que generan actividades para la conservación, protección y uso adecuado de los recursos genéticos forestales. (Grijalva et al, 2012).

Para ello es necesario la selección de especies forestales nativas en la propia región (Naula, 2013). La selección e identificación de árboles de alto rendimiento, es el inicio y la base fundamental de un programa de mejoramiento genético forestal la cual involucra a la generación de información del sitio donde se puede recolectar semilla (Vallejo, 2010).

El estudio pretende identificar y seleccionar árboles semilleros de especies forestales maderables, existente en las comunas de Olón, Dos Mangas y Salanguillo, con la finalidad que los productores de plantas en viveros dispongan de información sobre la existencia de árboles padres productores de semilla, seleccionados como padres. Dicha información será base para mejorar la genética de plantaciones maderables en la provincia de Santa Elena.

\subsection{Selección de fuentes semillas}

Las fuentes semilleros forestales son la base para implementar programas de mejoramiento genético forestal, por tener en claro las procedencias y fuentes de semillas, sitios seleccionados con altos porcentajes de árboles sanos y buen fenotipo. (Niembro, 1985), citado por (Meza Sandoval, 2014)

Los árboles semilleros son especies forestales padres que conservan características deseables para producir semillas, teniendo en claro la fenología de la especie y calendario de producción de semillas (University of California, 2007).

En el Ecuador las semillas forestales para la propagación de nuevas plantas, es poco analizada la procedencia a pesar de que son de suma importancia para la forestación y reforestación, esto se debe a la falta de cumplimiento de leyes (Prado et al, 2010).

Los métodos de selección de árboles semilleros para la reproducción natural de plantas es parte de un sistema silvicultural de mayor aplicabilidad en los países productores de madera. (Bolfor, 2003)

\section{Materiales y Métodos}

El proyecto de selección de árboles semilleros para la producción de semillas de especies forestales, se realizó desde mayo de 2016 a enero de 2017, en las comunas Olón, Dos Mangas y Salanguillo de la provincia de Santa Elena. La selección de árboles se realizó dentro de plantaciones forestales que presentan especies establecidas entre los años 2000 y 2005 por el Ministerio del Ambiente, en las comunas Olón y Dos Mangas pertenecen a la parroquia Manglaralto, ubicadas en un bosque seco tropical, altitud de 50 msnm, con temperatura media de $25^{\circ} \mathrm{C}$ y una precipitación de $600 \mathrm{~mm} /$ año con presencia de dos épocas de lluvia el invierno que se presenta en los meses de enero a abril y la garúa (presencia de pequeñas lloviznas) entre los meses de julio a noviembre; la comuna Salanguillo forma parte de la parroquia Colonche, ubicada en un tipo de bosque seco tropical 
con temperatura media de $25^{\circ} \mathrm{C}$ y $400 \mathrm{~mm} / \mathrm{año}$, altitud de $60 \mathrm{msnm}$ presencia de lluvias solamente en la temporada de invierno (enero a abril). Las comunas mencionadas forman parte de la cordillera Chongón Colonche, lugar donde se ubica el bosque protector Chongón Colonche área de gran diversidad de especies vegetales y animales.

\subsection{Identificación de plantaciones para selección de árboles semilleros}

En la comuna Olón se identificó un área 40 hectáreas plantada con diferentes especies de forestales entre las que destacan, laurel blanco (Cordia alliodora), laurel negro (Cordia megalantha), cedro (Cedrela odorata), caoba (Switenia macrophylla), melina (Gmelina arbórea), pechiche (Vitex gigantea), guayacán (Tabebuya crisantha), bálsamo (Myroxilum balsamun), Fernán Sánchez (Triplaris cumingiana), entre otras; densidad de plantación de 400 árboles/ha, y de una edad aproximada de 17 años.

En la comuna Dos Mangas se identificaron 3 fincas en las que identificó cedro $(C$. odorata), laurel blanco (C. alliodora), laurel negro (C. megalantha), caoba (S. macrphylla), guayacán (T. crisantha), pechiche (V. gigantea), entre otras; densidad de plantación de 400 árboles/ha, de una edad entre 12 a 17 años. Mientras que en la comuna Salanguillo se identificaron 2 fincas que presentan especies forestales similar a la comuna Dos Mangas, con una densidad de plantación de 400 árboles/ha, de una edad entre 12 a 17 años.

\subsection{Método de selección de árboles semilleros}

Para el efecto se realizó el recorrido de las fincas en el área forestal. Se utilizó cartografía básica de los lugares; GPS Garmin $72 \mathrm{H}$ de alta precisión para la georreferenciación de los árboles seleccionados y codificación, permitiendo identificar especies con características fenológicas deseables (Cerón y Sahagún, 2005). La selección de árboles semilleros en el estudio, se basó en los "parámetros de selección de Heredia y Hofstede (1999), adaptado por (Ordoñez, et al., 2001) Que considera las características dasonómicas y morfológicas individuales de los árboles semilleros; la ubicación de los árboles se enmarcó en el sistema de coordenadas UTM Datum WGS-1984 17M (Tabla 1).

\section{Resultados y Discusión}

En la tabla 1, se muestra las especies seleccionadas, así como, la codificación por especie y edad, la misma que fue entre 12 a 17 años, al respecto. (ZapataValenzuela y Hasbun, 2011) consideran que la selección de los fenotipos a temprana edad facilita el proceso, reduciendo etapas para lograr un material rápido y disponible para la industria
Una vez realizadas las mediciones se preseleccionó árboles de cinco especies laurel negro (Cordia megalantha) y blanco (Cordia alliodora L), Caoba (Swietenia macrophylla) Melina (G. arborea Roxb) y cedro (C. adorata).

Tabla 1. Selección de árboles semilleros

\begin{tabular}{|c|c|c|c|}
\hline \multirow{2}{*}{ Especie } & Comuna & $\begin{array}{c}\text { Código } \\
\text { de árbol }\end{array}$ & $\begin{array}{c}\text { Edad de } \\
\text { plantación }\end{array}$ \\
\hline \multirow{2}{*}{$\begin{array}{c}\text { Cordia } \\
\text { allidora }\end{array}$} & Olón & OJ13 & 17 años \\
\cline { 2 - 4 } & Salanguillo & SA08 & 12 años \\
\cline { 2 - 4 } & SE01 & 12 años \\
\hline \multirow{2}{*}{$\begin{array}{c}\text { Cordia } \\
\text { megalantha }\end{array}$} & Olón & OJ06 & 17 años \\
\cline { 2 - 4 } & Dos Mangas & JS04 & 12 años \\
\hline \multirow{2}{*}{$\begin{array}{c}\text { Gmelina } \\
\text { arbórea }\end{array}$} & Olón & OJ03 & 17 años \\
\hline \multirow{2}{*}{$\begin{array}{c}\text { Cedrela } \\
\text { odorata }\end{array}$} & Dos Mangas & MG02 & 17 años \\
\cline { 2 - 4 } $\begin{array}{c}\text { Swietenia } \\
\text { macrophylla }\end{array}$ & Salanguillo & SE04 & 17 años \\
\cline { 2 - 4 } & Salanguillo & SA04 & 17 años \\
\hline
\end{tabular}

Elaborado por: autores

\subsection{Características dasométricas:}

Las características dasométricas (Tabla 2), el diámetro a la altura del pecho (DAP), se midió a una altura de 1,30 $\mathrm{m}$ desde el nivel del suelo los promedios variaron de 20,3 centímetros para el laurel blanco ( $C$. allidora) de 12 años en la comuna Salanguillo y el valor mayor fue para cedro (C. adorata) de 17 años con 50 $\mathrm{cm}$ en la comuna Dos Mangas.

Altura total (HT): se valoró entre el nivel del suelo y el ápice del árbol, se utilizó un hipsómetro para su medición; el comportamiento de esa variable señala a la melina ( $G$. arbórea) con mayor altura entre las especies seleccionadas alcanzando 19 metros de igual manera destaca este árbol semillero en la variables relacionadas con altura fustal (HF) que se considera la distancia existente entre el nivel del suelo (base del árbol) hasta la primera bifurcación del árbol con valor de $10,25 \mathrm{~m}$ el menor valor fue para Caoba (C. odorata) con $2,8 \mathrm{~m}$ de 17 años en la comuna Salanguillo. En lo relacionado con la altura comercial (HC) se determinó entre la base del árbol y el punto del fuste hasta donde se considere comerciable, destacando melina (G. arborea Roxb) con $10,25 \mathrm{~m}$. 
Tabla 2. Dasometría de árboles seleccionados

\begin{tabular}{|c|c|c|c|c|c|}
\hline \multirow{2}{*}{$\begin{array}{c}\text { Código } \\
\text { de } \\
\text { árbol }\end{array}$} & Edad de & plantación & \multicolumn{4}{|c|}{ Características Dasométricas } \\
\cline { 3 - 6 } & $\begin{array}{c}\text { DAP } \\
(\mathbf{c m})\end{array}$ & $\begin{array}{c}\text { HT } \\
(\mathbf{m})\end{array}$ & $\begin{array}{c}\text { HF } \\
(\mathbf{m})\end{array}$ & $\begin{array}{c}\text { HC } \\
(\mathbf{m})\end{array}$ \\
\hline OJ13 & 17 años & 22,3 & 18 & 8 & 7 \\
\hline SA08 & 12 años & 20,3 & 13,5 & 6,9 & 6,9 \\
\hline SE01 & 12 años & 21 & 12 & 7,64 & 8,75 \\
\hline OJ06 & 17 años & 22,3 & 20 & 8,98 & 7,11 \\
\hline OJ07 & 17 años & 22,3 & 16 & 8,32 & 8,32 \\
\hline JS04 & 12 años & 22 & 17 & 6,75 & 8,27 \\
\hline OJ03 & 17 años & 31,2 & 19 & 10,25 & 10,25 \\
\hline MG02 & 17 años & 50 & 17 & 6,85 & 5,8 \\
\hline SE04 & 17 años & 25 & 12 & 2,8 & 6,9 \\
\hline CG02 & 12 años & 26 & 11 & 6 & 6 \\
\hline SA04 & 17 años & 23 & 12 & 4,99 & 4,99 \\
\hline
\end{tabular}

Elaborado por: autores

\subsection{Parámetros morfológicos utilizados en la selección de árboles semilleros}

La forma del fuste para su aprovechamiento maderable se ubica en recto y ligeramente torcido alcanzando valoración entre 6 y 4 respectivamente en todas las especies forestales. En altura de bifurcación el mejor comportamiento en esta valoración es para 6 árboles no bifurcados y los restantes con bifurcación en el tercio superior. La dominancia del eje principal se refiere a la forma del tallo principal, donde se considera la dominancia completa en el eje principal calificado sobre 2, o dominancia parcial sobre las ramas laterales de los obteniendo calificación de 1. En ángulo de rama se observa el ángulo de inserción de las ramas con respecto al fuste que va de $60^{\circ}$ a $90^{\circ}$ y se presenta en nueve de los once árboles forestales obteniendo ponderación de 3 puntos. En la forma de copa la evaluación muestra que 9 se comportan de manera similar en el orden de 5 correspondiente a circular irregular, la suma total y valoración de cada uno de los materiales alcanzan valores para ser seleccionados como árboles semilleros según la metodología adoptada en el estudio (Tabla 3).

Una vez realizada la valoración de los materiales en las plantaciones establecidas en las comunas, se identificaron y seleccionaron los mejores árboles en las plantaciones, tomando en consideración sus características morfológicas y dasométricas (Ordoñez, et al, 2004). Las valoraciones finales establecen a 11 árboles de cinco especies forestales con características de árboles semilleros (Tabla 4). La selección de árboles es el sistema que elige las mejores especies forestales para dar inicio a una población mejorada genéticamente (Ipinza, 1997)

Tabla 3. Características morfológicas de árboles seleccionados

\begin{tabular}{|c|c|c|c|c|c|c|}
\hline $\begin{array}{c}\text { Código } \\
\text { de } \\
\text { árbol }\end{array}$ & $\begin{array}{c}\boldsymbol{\emptyset} \\
\text { Copa } \\
(\mathbf{m})\end{array}$ & $\begin{array}{c}\text { HF } \\
(\mathbf{m})\end{array}$ & $\begin{array}{c}\text { HB } \\
(\mathbf{m})\end{array}$ & $\begin{array}{c}\text { Dominancia } \\
\text { del eje } \\
\text { principal }\end{array}$ & $\begin{array}{c}\text { Ang. } \\
\text { Rama }\end{array}$ & $\begin{array}{c}\text { F. } \\
\text { de } \\
\text { Copa }\end{array}$ \\
\hline OJ13 & 3 & 6 & 6 & 2 & 3 & 5 \\
\hline SA08 & 3 & 6 & 4 & 2 & 3 & 5 \\
\hline SE01 & 3 & 6 & 4 & 2 & 3 & 5 \\
\hline OJ06 & 3 & 6 & 6 & 2 & 3 & 5 \\
\hline OJ07 & 3 & 6 & 6 & 2 & 3 & 5 \\
\hline JS04 & 3 & 6 & 6 & 2 & 3 & 6 \\
\hline OJ03 & 3 & 6 & 6 & 2 & 3 & 5 \\
\hline MG02 & 7 & 4 & 4 & 1 & 3 & 5 \\
\hline SE04 & 3 & 6 & 4 & 2 & 3 & 5 \\
\hline CG02 & 3 & 6 & 6 & 1 & 2 & 5 \\
\hline SA04 & 3 & 6 & 4 & 2 & 2 & 6 \\
\hline
\end{tabular}

Elaborado por: autores

Tabla 4. Georreferenciación y valoración de parámetros morfológicos de Árboles semilleros

\begin{tabular}{|c|c|c|c|}
\hline $\begin{array}{l}\text { Código } \\
\text { de } \\
\text { árbol }\end{array}$ & $\begin{array}{c}\text { Coordenadas } \\
\text { UTM } \\
\text { WGS 1984 } \\
\text { 17M } \\
\end{array}$ & $\begin{array}{c}\text { Suma } \\
\text { parámetros } \\
\text { Morfológicos }\end{array}$ & Valorización \\
\hline OJ13 & $\begin{array}{c}9785435 \mathrm{~N}- \\
524765 \mathrm{E}\end{array}$ & 25 & 1 \\
\hline SA08 & $\begin{array}{c}9780834 \mathrm{~N}- \\
548589 \mathrm{E} \\
\end{array}$ & 23 & 1 \\
\hline SE01 & $\begin{array}{c}9780570 \mathrm{~N}- \\
548596 \mathrm{E}\end{array}$ & 23 & 1 \\
\hline OJ06 & $\begin{array}{c}9801653 \mathrm{~N}- \\
529117 \mathrm{E} \\
\end{array}$ & 25 & 1 \\
\hline OJ07 & $\begin{array}{c}9801659 \mathrm{~N}- \\
529012 \mathrm{E}\end{array}$ & 25 & 1 \\
\hline JS04 & $\begin{array}{c}9798737 \mathrm{~N}- \\
535944 \mathrm{E}\end{array}$ & 26 & 1 \\
\hline OJ03 & $\begin{array}{c}9801668 \mathrm{~N}- \\
529275 \mathrm{E}\end{array}$ & 25 & 1 \\
\hline MG02 & $\begin{array}{c}9799472 \mathrm{~N}- \\
536268 \mathrm{E}\end{array}$ & 24 & 1 \\
\hline SE04 & $\begin{array}{c}9780605 \mathrm{~N}- \\
548546 \mathrm{E}\end{array}$ & 23 & 1 \\
\hline CG02 & $\begin{array}{c}9799758 \mathrm{~N}- \\
535640 \mathrm{E}\end{array}$ & 23 & 1 \\
\hline SA04 & $\begin{array}{c}9780823 \mathrm{~N}- \\
548626 \mathrm{E}\end{array}$ & 23 & 1 \\
\hline
\end{tabular}

Elaborado por: autores 


\subsection{Producción de semilla}

La estimación de producción de semilla por árbol y especie seleccionada (Tabla 5), se pondera el rendimiento teórico promedio para cada especie considerando el porcentaje germinativo de $60 \%$ (MAGAP, 2012) con el que se obtiene una producción considerable de plantas que se puede utilizar como guía para la elaboración y ejecución de proyectos de forestación y reforestación en el bosque seco peninsular ecuatoriano.

Tabla 5. Estimación mínima de producción de semillas y plántulas por año

\begin{tabular}{|l|c|c|c|c|}
\hline \multicolumn{1}{|c|}{ Especie } & $\begin{array}{c}\text { Árboles } \\
\text { seleccionados }\end{array}$ & $\begin{array}{c}\text { Kilos } \\
\text { de } \\
\text { semilla } \\
\text { /árbol } \\
\text { /año }\end{array}$ & $\begin{array}{c}\text { semillas } \\
\text { /kilo }\end{array}$ & $\begin{array}{c}\text { Plantas } \\
\text { año } \\
(\mathbf{6 0 \%})\end{array}$ \\
\hline $\begin{array}{l}\text { Cordia } \\
\text { allidora }\end{array}$ & 3 & 1 a 2 & 20000 & 36000 \\
\hline $\begin{array}{l}\text { Cordia } \\
\text { megalantha }\end{array}$ & 3 & 2 a 3 & 10000 & 12000 \\
\hline $\begin{array}{l}\text { Gmelina } \\
\text { arbórea }\end{array}$ & 1 & 4 a 6 & 1000 & 2400 \\
\hline $\begin{array}{l}\text { Cedrela } \\
\text { odorata }\end{array}$ & 2 & 1 a 2,5 & 4000 & 4800 \\
\hline $\begin{array}{l}\text { Swietenia } \\
\text { macrophylla }\end{array}$ & 2 & 2 a 4 & 1300 & 1560 \\
\hline
\end{tabular}

\section{Conclusiones}

La selección de árboles semilleros es el inicio para disponer de semillas procedentes de árboles con características deseables para la producción de plantas y lograr plantaciones forestales maderables con materiales propios de la provincia de Santa Elena.

Las especies forestales seleccionadas corresponde a Cordia alliodora, Cordia megalantha, Cedrela adorata, Gmelina arbórea y Swietenia macrophylla. De estas especies, 11 árboles cumplen con características sobresalientes para ser considerados árboles padres o semilleros, en edades comprendidas entre 12 y 17 años, que garantiza una buena producción de semillas en el tiempo.

La cantidad de semillas proyectada por año servirá como referencia para establecer programas de forestación y reforestación que se ejecuten en la provincia y el país.

\section{Referencias}

[1] Bolfor, 2003. Consideraciones para árbol semillero en bosques tropicales bajo manejo en Bolivia: Proyecto BOLFOR-The Forest Management Trust,. Santa Cruz de la Sirra, Bolivia: s.n.
[2] Cerón, R. \& Sahagún, J., 2005. Un índice de seleción basado en componentes principales. Red de Revistas Científicas de America Latina, El Caribe, España y Portugal, Volumen 39, pp. $667-$ 677.

[3] CIIFEN, 2014. Conservación y manejo de recursos naturales, Proyecto: Enfrentando el cambio climático en la cordillera Costera, CentroInternacional para la Investigación del Fenómeno El Niño,. Guayaquil, Ecuador: s.n.

[4] Grijalva, j. y otros, 2012. Situación de los Recursos Genéticos Forestales-Informe País Ecuador, Quito: s.n.

[5] Grijalva, J., Ramos, R., Barrera, P. \& Sigcha, F., 2013. Primer Encuentro Nacional de Bosques, Recursos Genéticos Forestales y Agroforesteria. Memorias del Evento. Instituto de investigaciones agropecuarias.. Quito, Ecuador: s.n.

[6] Ipinza, R., 1997. Acta 1er Taller de mejora genética r Roble y Raulí, Aspectos teóricos en la selección de parboles superiore. Valdivi, Chile: s.n.

[7] Lojan, L., 2003. El verdor de los Andes Ecuatorianos: realidades y promesas. s.1.:SOBOC.

[8] MAE., 2014. Plan Nacional de Restauración Forestal 2014-2017. p. 50.

[9] MAE, 2013. Sistema de Nacional de Control Forestal, Quito: s.n.

[10] MAGAP, M. d. A. G. y. P., 2012. Ecuaforestal Ficha Técnica $\mathrm{N}^{\circ}$ 4. Laurel blanco. [En línea] Available at: http://ecuadorforestal.org/fichastecnicas-de-especies-forestales/ficha-tecnica-no-4laurel/

[Último acceso: 4 mayo 2017].

[11] Meza Sandoval, B. E., 2014. Identificación y Selección de Árboles plus de las especies Tabebuia donnell-smithii Rose y Tabebuia rosea Bertol en el Soconusco. Chiapas: s.n.

[12] Morandini, R., s.f. Fao.org. [En línea] Available at: http://www.fao.org/docrep/03650s/03650s00.htm\# Contents

[Último acceso: 28 Septiembre 2016]. 
[13] Naula, J., 2013. Las Plantas Nativas. [En línea] Available at:

http://lasplantasnativas800.blogspot.com/2013/04/p lantas-nativas-plantas-nativas-son-

las.html\#comment-form

[Último acceso: Noviembre 2016].

[14] Ordoñez, L., Aguirre, N. \& Hofstede, R., 2001. Sitios de recolección de semillas Forestales andinas del Ecuador. Quito: Ecopar.

[15] Ordoñez, L., Arbeláez, M. V. \& Prado , L., 2004. Manejo de semillas forestales nativas de la sierra del Ecuador y Norte del Perú. Quito: EcoPar.

[16] Prado, L., Samaniego , C. \& Ugarte-Guerra, J., 2010. Estudio de las cadenas de abastecimiento de germoplasma forestal en Ecuador. World Agroforestry Centre, p. 115.

[17] University of California , 2007. Forest Vegetation Management. Oakland(California): s.n.
[18] Vallejo, e. a., 2010. Metodología para la selección e incorporación de árboles plus en programas de mejoramiento genético forestal. [En línea]

Available at:

http://www.scielo.sa.cr/scielo.php?script=sci_artte xt\&pid=S0377-94242010000100011

[Último acceso: 24 Abril 2017].

[19] Viteri, A., 2010. Documento de análisis del sector forestal en el contexto de adaptación y mitigación al cambio clamático del sector uso del suelo, cambio de suelo y silvicultura (forestal) en el Ecuador. Quito: s.n.

[20] Zapata-Valenzuela, J. \& Hasbun, R., 2011. Mejoramiento genético forestal acelerado mediante selección genómica. Genómica Forestal. 